\title{
Mass Transfer in Binary Stellar Evolution and Its Stability
}

\author{
Seblu Humne Negu, Solomon Belay Tessema \\ Astronomy and Astrophysics Research Division, Entoto Observatory and Research Center (EORC), \\ Addis Ababa, Ethiopia \\ Email: seblu1557@gmail.com, tessemabelay@gmail.com
}

Received 30 May 2015; accepted 27 September 2015; published 30 September 2015

Copyright (C) 2015 by authors and Scientific Research Publishing Inc.

This work is licensed under the Creative Commons Attribution International License (CC BY). http://creativecommons.org/licenses/by/4.0/

c) (i) Open Access

\begin{abstract}
The evolution of a binary star system by various analytical and numerical approximations of mass transfer rate normalized to the equilibrium rate and its stability conditions are investigated. We present results from investigations of mass transfer and stability in close binary star systems using the different orbital parameters. The stability and instability of mass transfer in binary star evolution depends on the exchange of material which the response of the binary to the initial Roche lobe overflow causes the donor to loose even more material. Our work is mainly focused on basic mathematical derivations, analytical and numerical solutions in order to explain the mass transfer system in different orbital parameters as well as the results are compared with previous studies in both cases. Mass transfer is usually stable, as long as the winds specific angular momentum does not exceed the angular momentum per reduced mass of the system. This holds for both dynamical and thermal time scales. Those systems which are not stable will usually transfer mass on the thermal time scale. The variation of Roche lobe radius with mass ratio in the binary, for various orbital parameters in the conservative and non-conservative mass transfer, as well as the evolution equations, orbital angular momentum of the binary system and the corresponding analytical and numerical solutions for different cases, under certain restrictive approximations is derived, simulated and discussed.
\end{abstract}

\section{Keywords}

Mass Transfer, Binary Star, Stellar Evolution, Stability, Instability

\section{Introduction}

The dominant feature in the evolution of stars is in closed binaries, and the one which distinguishes it from the evolution of single stars is the presence of various forms of mass transfer between the two stars. Mass transfer 
occurs in many different types of systems, to widely varying effects [1]. Contamination of the envelope of a less evolved star with chemically processed elements, as in Barium stars [2], and catastrophic mass transfer, by common envelope phase, as in W UMa systems; or a slow, steady mass transfer by Roche lobe overflow [2].

Close binary stars consisting of two compact stellar remnants (white dwarfs (WDs), neutron stars (NSs), or black holes (BHs)) are considered as primary targets of the forthcoming field of gravitational wave $(G W)$ astronomy [3] since their orbital evolution has entirely controlled by the emission of gravitational waves and leads to ultimate coalescence (merger) of the components. GW emission is the sole factor responsible for the change of orbital parameters of a pair of compact (degenerate) stars. However, at the early stages of binary evolution, it is the mass transfer between the components and the loss of matter and its orbital angular momentum that play a dominant dynamical role.

Mass transfer is particularly interesting if one considers the evolution of a system with at least one degenerate star. In these cases, mass transfer produces spectacular effects, resulting in part from the intense magnetic and gravitational fields of the compact objects, pulsed X-ray emission, nuclear burning, novae outbursts, and so on. Also, since the mass transfer rates can be high and orbital period measurements accurate, one may see the dynamical effects of mass transfer on the binary orbit, as in Cygnus X-3 [4].

In the case of cataclysmic variables (CVs) and low mass X-ray binary system (LMXBs), one has a highly evolved, compact star (CVs and LMXBs contain white dwarfs and neutron stars, respectively) and a less evolved main sequence or red giant star. Mass transfer usually proceeds by accretion onto the compact object, and is secularly stable. Most of these objects involve one component filling its Roche lobe (the donor or secondary), transferring mass to a compact star (the accretor or primary) and the mass leaking out of the inner Lagrangian point forms an accretion disk around the accretor. A binary system starts out as detached [4] [5], with both stars in hydrostatic equilibrium and filling an equipotential surface inside their Roche lobes. In a binary, both stars $(\odot 1$ and $\odot 2$ can fill their Roche lobes at subsequent evolutionary stages). We use the convention which $\odot 1$ denotes the primary star, and $\odot 2$ denotes the secondary star of the binary. In this convention, the mass ratio is $q=m_{2} / m_{1}=m_{d} / m_{a}>1$. During phases of mass transfer, we denote the mass-losing star, the "donor", with subscript " $d$ " and the companion star, the "accretor", with subscript " $a$ ". The transfer will be accompanied by a stellar wind from the mass-losing star, or ejection of matter from the accretor, as in novae and galactic jet sources.

There are various unanswered questions in the evolution of LMXBs into low mass binary pulsars (LMBPs, in which a millisecond radio pulsar is in a binary with a low mass white dwarf companion) and of CVs. Among these are the problem of the disparate birthrates of the LMXBs and the LMBPs (Kulkarni and Narayan1988), estimation of the strength of X-ray heating induced winds from the donor star.

Now, if a star is a member of a binary star system, then it is possible that as the components evolving, one of them (or both) can fill up its Roche lobe. Thomas (1977) classified mass transferring binaries on the basis of what state of evolution the donor is in as follows:

Case A: If the orbital separation is small enough (usually a few days), the star can fill its Roche lobe during its slow expansion through the main-sequence phase while still burning hydrogen in its core.

Case B: If the orbital period is less than about 100 days, but longer than a few days, the star will fill its Roche lobe during the rapid expansion to a red giant with a helium core. If the helium core ignites during this phase and the transfer is interrupted, the mass transfer is Case B.

Case C: If the orbital period is above 100 days, the star can evolve to the red supergiant phase before it fills its Roche lobe. In this case, the star may have a CO or ONe core.

Case A mass transfer occurs during the slow growth, Case B during the first rapid expansion, and Case $\mathrm{C} \mathrm{du-}$ ring the final expansion phase. The nature of the remnant depends upon the state of the primary during the onset of RLOF and the orbital properties of the resultant binary depend upon the details of the mass transfer.

The problem of mass transfer in binaries by Roche lobe overflow has received a good deal of attention in the literature over the past few decades, typically in investigations of one aspect or another of orbital evolution or stability. [6] examined mass transfer by isotropic winds and accretion, in investigating the evolution of short period binary X-ray sources with extreme mass ratios, and developed models with accretion and (typically) isotropic re-emission of transferred matter, in the context of the period gap in cataclysmic variables.

Stability has become an important in many area of astrophysical research. Many authors has been studied the stability in different models (for example, [7] [8]). Both authers didn't address stability and mass transfer in binary stellar evolution. [1] [2] [4] were tried to study stability criteria for mass transfer in binary stellar evolution using limits of pure models of mass transfer and extreme mass ratio. The present study will address the mass 
transfer in binary stellar evolution using analytical and numerical methods which will give brief relations about stability and mass transfer.

The aim of this paper is to study mass transfer in binary stellar evolution behavior using mass transfer and different orbital parameters. This will be used to study stability properties of binary stars using analytical and numerical solutions. We calculate the rate of change of orbital angular momentum, orbital periods, angular speed and stability analysis of mass transfer rates, with relevant evolution in general form.

The paper is organized as follows:

In Section (2), we derive basic equations of mass transfer, mass transfer and evolution of orbital parameters, conservative and non-conservative mass transfer and evolution equations; in Section (3), stability analysis of mass transfer and its rate will be determined; in Section (4), analytical and numerical solutions will be presented. Finally our main conclusions are summarized in Section (5).

\section{Basic Equations of Mass Transfer}

\subsection{Mass Transfer and the Evolution of Orbital Parameters}

In this section, expressions for the variation of orbital parameters with loss of mass from one of the stars are derived. In what follows, the two stars will be referred to as $m_{1}$ and $m_{2}$, with the latter the mass losing star.

The angular momentum (AM) of binary component $i$ in a circular orbit is expressed as:

$$
J_{i}=\left\|\mathrm{J}_{i}\right\|=m_{i}\left\|\mathrm{v}_{i} \times \mathrm{a}_{i}\right\|=m_{i} v_{i} a_{i}
$$

where $v_{i}=\omega a_{i}$ is the velocity of accretor and donor star; " $i$ " stands for the accretor " $a$ " and donor " $d$ " stars respectively, $a_{i}$ is the position of the accretor and donor star, and $\omega$ is the orbital angular spin frequency.

$$
J_{i}=m_{i} a_{i}^{2} \omega
$$
by

Roche geometry changes are governed by the total orbital angular momentum $J$ of the system which is given

$$
J=J_{a}+J_{d}=\left(m_{a} a_{1}^{2}+m_{d} a_{2}^{2}\right)=\mu a^{2} \omega=\mu \sqrt{G M_{T} a}
$$

where $a_{a}=\left(m_{d} / M_{T}\right) a, a_{d}=\left(m_{a} / M_{T}\right) a$ are the distance of the two stars from the center of mass (semi major-axis), $\mu=m_{a} m_{d} /\left(m_{a}+m_{d}\right)$ is the reduced mass, $M_{T}=m_{a}+m_{d}$ is the total mass, and the mass ratio is $q=m_{d} / m_{a}$. Throughout this paper, we consider an eccentricity $e$ will be zero.

The period "P", the semi major axis " $a$ " and the total mass transfer related through Kepler's law as:

$$
(2 \pi / P)^{2}=\omega^{2}=\frac{G M_{T}}{a^{3}}
$$

which implies that the orbital angular momentum $J$ can be written as:

$$
J(P)=m_{a} m_{d}\left(\frac{P G^{2}}{2 \pi M_{T}}\right)
$$

The angular momentum for binary component " $i$ ” is given by

$$
J_{i}=\frac{\mu J}{m_{i}}
$$

and the specific angular momentum for binary component “ $i$ ” can be written as:

$$
l_{i}=J_{i} / m_{i}=\mu \mathrm{J} / m_{i}^{2}
$$

where $m_{i}$ is mass of the accretor and donor and $l_{i}$ is specific angular momentum of the accretor and donor star, respectively.

From Kepler's $3^{\text {rd }}$ we relate orbital evolution as: 


$$
a^{3} \propto P^{2} M \sim 3 \frac{\dot{a}}{a}=2 \frac{\dot{P}}{P}+\frac{\dot{M}}{M}
$$

Hence from Equation (4) the orbital angular momentum $J$ of a binary star is given by

$$
J=m_{a} m_{d}\left(\frac{G a\left(1-e^{2}\right)}{M}\right)^{1 / 2}
$$

In many cases the angular momentum stored in the rotation of the two stars is negligible compared to the orbital angular momentum, so that Equation (8) also represents the orbital angular momentum of the binary, to good approximation. By differentiating this expression we obtain a general equation for the evolution of orbital parameters:

$$
\frac{\dot{J}}{J}=\frac{\dot{m}_{a}}{m_{a}}+\frac{\dot{m}_{d}}{m_{d}}=\frac{1}{2} \frac{\dot{a}}{a}-\frac{1}{2} \frac{\dot{M}}{M}-\frac{1}{2} \frac{2 e \dot{e}}{\left(1-e^{2}\right)}
$$

and

$$
\frac{\dot{a}}{a}=\frac{\dot{M}}{M}+2 \frac{\dot{J}}{J}-2\left(\frac{\dot{m}_{a}}{m_{a}}+\frac{\dot{m}_{d}}{m_{d}}\right)-\frac{e \dot{e}}{\left(1-e^{2}\right)}
$$

In Equation (10), $e$ is a parameter that determines the amount by which its orbit around another body deviates from a perfect circle. From Equation (7) and Equation (10) the rate of change of orbital period, semi major axis, and total mass transfer can be written as:

$$
\frac{\dot{P}}{P}=\frac{3}{2} \frac{\dot{a}}{a}-\frac{1}{2} \frac{\dot{M}}{M}
$$

or

$$
\frac{\mathrm{d} p}{p \mathrm{~d} t}=\frac{3}{2} \frac{\mathrm{d} a}{a \mathrm{~d} t}-\frac{1}{2} \frac{\mathrm{d} M}{M \mathrm{~d} t}
$$

which depends on time “t”.

By integrating Equation (11) we obtain:

$$
P=P_{0} \sqrt{\left(\frac{a}{a_{0}}\right)^{3}\left(\frac{M_{0}}{M}\right)}
$$

where $P_{0}, a_{0}$, and $M_{0}$ are initial orbital period, semi major axis, and total mass transfer of the binary stars, respectively.

In the case of Roche lobe overflow in an already circularized binary, the last term is zero. The $j$ term represents angular momentum loss from the binary, which can be due to spontaneous processes (such as gravitational wave radiation) or it can be associated with mass loss from the binary as a whole or from the component stars.

\subsection{Conservative Mass Transfer}

When no ejected matter leaves a binary system, the mass transfer is said to be conservative. During conservative mass transfer, the orbital elements of the binary can change due to transfer of angular momentum from one star to the companion. Consider a system with a total mass $M=m_{a}+m_{d}$, semi-major axis, $a$, eccentricity, $e$, and the total orbital angular momentum, $J$ is given by

$$
J=m_{a} m_{d} \sqrt{\frac{G a\left(1-e^{2}\right)}{m_{a} m_{d}}}
$$


will also be conserved, where $G$, is universal gravitational constant.

We first consider conservative mass transfer, in which the total mass and orbital angular momentum of the binary are conserved. In that case we can set as:

In circular, $e=\dot{e}=0$ and conservative mass exchange, $\dot{M}=\dot{J}=0$ and $m_{a}=-m_{d}>0$, then from Equation (13) we have:

$$
a=C^{2}\left(m_{a} m_{d}\right)^{-2} J^{2}
$$

where $C=\sqrt{M / G\left(1-e^{2}\right)}$, is a constant. Hence from Equation (10) we obtain:

$$
\frac{\dot{a}}{a}=-2\left(\frac{-\dot{m}_{d}}{m_{a}}+\frac{\dot{m}_{d}}{m_{d}}\right)=2 \frac{\dot{m}_{d}}{m_{d}}(q-1)
$$

Using the Kepler's third law in Equation (15), the period variation with time $\dot{P}$ due to mass transfer can be written as:

$$
\frac{\dot{P}}{P}=\frac{3}{2} \frac{\dot{a}}{a}
$$

and integrating Equation (16) which leads us

$$
P=P_{0} \sqrt{\left(\frac{a}{a_{0}}\right)^{3}}
$$

An explicit relation between the separation and the masses can be found by integrating Equation (15) with $e=0 ; m_{d}^{2} m_{a}^{2} a=$ constant.

Applying Kepler's law we obtain similar relation between the rate of change of the orbital period $\dot{P}$ and the rate of mass transfer $\dot{m}_{d}$, and between the period and masses directly from Equation (15):

$$
\frac{\dot{P}}{P}=3(q-1) \frac{\dot{m}_{d}}{m_{d}}=3 \frac{\dot{m}_{a}\left(m_{a}-m_{d}\right)}{m_{a} m_{d}}
$$

The importance of Equation (15) and Equation (18) is that it allows to determine the mass transfer rate of observed semi-detached binaries, if the masses and the period derivative can be measured. This is complicated by the fact that many binaries show short-term period fluctuations, while for the long-term average of the period derivative, the trend has been determined with reasonable conditions as follows:

If $q<1, \dot{a}>0$, mass transfer from the less massive star to the more massive one will cause the orbit to enlarge.

If $q>1, \dot{a}<0$, mass transfer from the more massive star to the less massive one will cause the orbit to shrink.

\subsection{Non-Conservative Mass Transfer}

In case of non-conservative mass transfer both mass and angular momentum can be removed from the system. Following [9] Orbital Angular Momentum (OAM) of a two body system is given by

$$
J=\left(\frac{m_{a} m_{d}}{m_{a}+m_{d}}\right) a^{2} \Omega=\left(\frac{q}{(1+q)^{2}}\right) M a^{2}
$$

where $\left(\frac{m_{a} m_{d}}{m_{a} m_{d}}\right) a^{2}=I$ is moment of inertia, and $\Omega=\frac{2 \pi}{P}$ is the angular frequency of the binary.

Assuming isotropic mass loss from the surface of the components, then, we have the rate of angular momentum:

$$
\dot{J}=\left(\frac{q}{(1+q)^{2}}\right) \dot{M} a^{2} \Omega .
$$


Since the dynamics of a two body system obey Kepler's third law therefore one expects transfer of mass would change the, $a, P$ and $M$ accordingly

$$
3 \frac{\dot{a}}{a}+2 \frac{\dot{\Omega}}{\Omega}=\frac{\dot{M}}{M} \Rightarrow 3 \frac{\dot{a}}{a}+2 \frac{\dot{\omega}}{\omega}=\frac{\dot{M}}{M}
$$

Assume $q$ as a constant, differentiating Equation (19) with respect to time gives:

$$
\frac{\dot{J}}{J}=\frac{\dot{M}}{M}+2 \frac{\dot{a}}{a}+\frac{\dot{\Omega}}{\Omega}
$$

Substituting Equation (21) into Equation (22) we get the following two equations:

$$
\frac{\dot{J}}{J}=\frac{5}{3} \frac{\dot{M}}{M}-\frac{1}{3} \frac{\dot{\Omega}}{\Omega}=\frac{5}{3} \frac{\dot{M}}{M}+\frac{1}{3} \frac{\dot{P}}{P}
$$

and

$$
\frac{\dot{J}}{J}=\frac{3}{2} \frac{\dot{M}}{M}+\frac{1}{2} \frac{\dot{a}}{a}
$$

Therefore, the two equations tell us how the loss of OAM and mass are caused the orbital period, $P$, and orbital radius, $a$, to change. The isotropic mass loss implies:

$$
\dot{J}=\frac{\dot{M}}{M} J
$$

This equation implies that the only source of OAM loss is mass loss, which were assumed to be isotropic. Substituting $\dot{J} / J$ from equation Equation (25) into Equation (23) will give:

$$
\dot{P}=-2 \frac{\dot{M}}{M} P
$$

In Equation (26), since the $\dot{P} / P$ is measurable observationally, therefore, $\dot{M} / M$ can be calculated from Equation (26).

Now if we use the following equations taken from Stepien (1995) to calculate relative angular momentum lost from a system as:

$$
\begin{gathered}
J=G^{2 / 3} M^{5 / 3} \Omega^{-1 / 3} q(1+q)^{-2} \\
J_{\text {lost }}=\dot{J}=-\frac{1}{3} G^{2 / 3} M^{5 / 3} \Omega^{-4 / 3} q(1+q)^{-2} \dot{\Omega}
\end{gathered}
$$

and hence the relative OAM lost only by magnetized star wind:

$$
\frac{J_{\text {lost }}}{J}=-\frac{1}{3} \frac{\dot{\Omega}}{\Omega}
$$

where

$$
\dot{\Omega}=1.8 \times 10^{-8}\left[\frac{k^{2}\left(m_{a} R_{a}^{2}+m_{d} R_{d}^{2}\right)(1+q)^{2}}{q G^{2 / 3} M^{5 / 3}}\right] \Omega^{7 / 3} \mathrm{e}^{-1.3 \Omega}
$$

This equation (Equation (30)) tells us the total rate of change of the orbital angular frequency of the binary related to the mass lost from the system.

\subsection{Evolution Equations}

Now we have to enumerate some of the physical mechanisms that can influence the evolution of a binary, we 
can proceed to derive mathematical expressions in order to quantify these effects. To reiterate, we work under the following assumptions:

i) Roche potential describes the gravitational field;

ii) Kepler's laws are valid, and that the stars orbit around their common center of mass in circular orbits;

iii) the spin and orbit axes are all parallel to one another and;

iv) tidal effects are included even though we ignore the effects of the distortion of the stars, i.e. we assume spherically symmetric stars.

Now, the total angular momentum of the system is given by

$$
J_{\text {tot }}=J_{\text {orb }}+J_{a}+J_{d}=\mu a^{2} \Omega+K_{a} m_{a} R_{a}^{2} \omega_{a}+K_{d} m_{d} R_{d}^{2} \omega_{d}
$$

where $k_{i}$ is dimensionless constants depending on the internal structure of the the angular spin frequencies $\left(\omega_{i}\right)$, and $\left(J_{a}, J_{d}\right)$ are the spins of the accretor and donor respectively. The first term in Equation (31) represents the orbital angular momentum of the components, and the two other terms represent the spin angular momenta of the stars.

$$
J_{\text {orb }}=J_{\text {tot }}-\left(J_{a}+J_{d}\right)
$$

The rate of change of the spin momenta can be given as the sum of a consequential angular momentum term and a tidal term as:

$$
\dot{J}_{i}=\dot{m}_{i} l_{i}+\dot{J}_{i, t i d}
$$

where, $l_{a}$ and $l_{d}$ indicate the specific angular momenta of the matter arriving at the accretor and the matter leaving the donor respectively.

The second term in the Equation (33) represents the tidal term, which is a function of the degree of $\left(\Omega-\omega_{i}\right)$ and the tidal time scale $\left(\tau_{s_{i}}\right)$ is given by

$$
\dot{J}_{i, t i d}=\frac{k_{i m_{i} R_{i}^{2}}}{\tau_{s_{i}}}\left(\Omega-\omega_{i}\right)
$$

Substituting Equation (33) and Equation (34) into Equation (31).

$$
\dot{J}_{\text {orb }}+J_{s y s}+\dot{m}_{d}\left(l_{a}-l_{d}\right)-\frac{k_{a} m_{a} R_{a}^{2}}{\tau_{s_{i}}}\left(\Omega-\omega_{a}\right)-\frac{k_{d} m_{d} R_{d}^{2}}{\tau_{s_{i}}}\left(\Omega-\omega_{d}\right)
$$

where $J_{\text {orb }}=m_{a} m_{d}(G a / M)^{1 / 2}$, then we have $(\dot{J} / J)_{\text {orb }}=\frac{m_{d}}{m_{d}}(1-q)+\frac{1}{2} \frac{\dot{a}}{a}$.

Accordingly, from Equation (35) the rate of change of the orbital separation is given by

$$
\frac{\dot{a}}{2 a}=\frac{\dot{J}_{\text {sys }}}{J_{\text {orb }}}-\frac{m_{d}}{m_{d}}\left(1-q-m_{d} \frac{l_{a}-l_{d}}{j_{\text {orb }}}\right)-\frac{k_{a} m_{a} R_{a}^{2}}{J_{\text {orb }} \tau_{s_{a}}}\left(\Omega-\omega_{a}\right)-\frac{k_{d} m_{d} R_{d}^{2}}{J_{d} \tau_{s_{d}}}\left(\Omega-\omega_{d}\right)
$$

which we re-write for the convenience as:

$$
\begin{gathered}
\frac{\dot{a}}{2 a}=\frac{\dot{J}_{\text {sys }}}{J_{\text {orb }}}-\frac{\dot{J}_{a, t i d}+\dot{J}_{d, \text { tid }}}{J_{\text {orb }}}-\frac{\dot{m}_{d}}{m_{d}}\left(q_{a}-q\right) \\
q_{a}=1-m_{d} \frac{l_{a}-l_{d}}{J_{\text {orb }}}
\end{gathered}
$$

The sign of the terms on the right hand side of Equation (37) determines if the orbit is shrinking or expanding. $q_{a}$ represents the net effect of the consequential transfer of angular momentum. In the absence of mass transfer, the orbit shrinks as a result of the systemic angular momentum loss. 
If $q>q_{a}$ then the binary continues to shrink. On the other hand, for systems with $q<q_{a}$, mass transfer leads to the orbit expanding or least not shrinking as rapidly as it would in the absence of mass transfer.

The tidal terms have a more complicated behavior because the sign of the tidal torque $J_{i, \text { tid }}$ is a function of the difference in the spin frequencies and the orbital frequency, $\Omega-\omega_{i}$. If the spin frequency of a given component is higher than the orbital frequency, tides will tend to pump angular momentum into the orbit and thus help increase the separation. Conversely, if the orbital frequency is higher than the spins, it can lead to a runaway instability since the tides will tend to suck more angular momentum out from the orbit which in turn will increase the orbital frequency even more.

We now determine how the depth of contact evolves to complete the set of equations we need to specify the evolution of the binary system. We can write equation of the Roche lobe size as:

$$
\frac{\dot{R}_{L}}{R_{L}}=\zeta_{r L} \frac{\dot{m}_{d}}{m_{d}}+\frac{\dot{a}}{a}
$$

where $R_{L}, \dot{R}_{L}$ are the Roche lobe radius and the rate of change of the Roche Lobe radius and $\zeta_{r L} \approx 1 / 3$ is the logarithmic derivative of $r L$ with respect to $m_{d}^{2}$. Thus we have:

$$
\frac{\dot{R}_{L}}{R_{L}}=2 \frac{\dot{J}_{\text {sys }}}{J_{\text {orb }}}-2 \frac{\dot{J}_{a, t i d}+\dot{J}_{d, t i d}}{J_{\text {orb }}}-2 \frac{\dot{m}_{d}}{m_{d}}\left(q_{a}-\frac{\zeta_{r L}}{2}-q\right)
$$

Symbolically, generalizing the meaning of the symbols introduced by [5]

$$
\begin{gathered}
\frac{\dot{R}_{L}}{R_{L}}=v_{L}+\zeta_{L} \frac{\dot{m}_{d}}{m_{d}} \\
v_{L}=2 \frac{\dot{J}_{\text {sys }}}{J_{\text {orb }}}-2 \frac{\dot{J}_{a, t i d}+\dot{J}_{d, t i d}}{J_{\text {orb }}} \\
\zeta_{L}=\zeta_{r L}+2\left(q-q_{a}\right)
\end{gathered}
$$

where the symbol $v_{L}$ stands for driving terms and $\zeta_{L}$ denotes logarithmic derivatives with respect to donor mass. We write the logarithmic time derivative of the donor radius $R_{d} \equiv R_{d}\left(m_{d}, t\right)$ as:

$$
\frac{\dot{R}_{d}}{R_{d}}=v_{d}+\zeta_{d} \frac{\dot{m}_{d}}{m_{d}}
$$

where $v_{d}$ represents the rate of change of the donor radius due to intrinsic processes such as thermal relaxation and nuclear evolution, whereas $\zeta_{d}$ usually describes changes resulting from adiabatic variations of $m_{d}$ as described in Equation (44). We derive here a simple analytic approximation to the effective mass-radius exponent when the response of the donor is a combination of the adiabatic and thermal adjustments to mass loss. from Equation (44).

$$
\frac{\dot{R}_{d}}{R_{d}}=\zeta_{d} \frac{\dot{m}_{d}}{m_{d}}=v_{d}+\zeta_{s} \frac{\dot{m}_{d}}{m_{d}}
$$

where $\zeta_{d}$ is the effective mass-radius exponent and $v_{d}$ stands for the thermal radial reaction rate, and $\zeta_{s}$ is here the purely adiabatic mass-radius exponent. As a consequence of mass loss, the donors radius $R_{d}$ will differ from the equilibrium radius corresponding to its instantaneous mass $R_{e q}\left(m_{d}\right)$. With these definitions we write:

$$
\frac{\dot{R}_{d}}{R_{d}}=\frac{\operatorname{Re} q\left(m_{d}\right)-R_{d}}{R_{d} \tau}+\zeta_{s} \frac{\dot{m}_{d}}{m_{d}}
$$

The secular evolution of the binary takes place on the mass transfer time scale $\tau_{m_{d}}=-\frac{m_{d}}{\dot{m}_{d}}$. Differentiating 
Equation (46) with respect to time, we get:

$$
\frac{\mathrm{d}^{2} \ln R_{d}}{\mathrm{~d} t^{2}}=\frac{1}{\tau} \frac{R_{e q}}{R_{d}}\left(\frac{\zeta_{e q}}{\tau_{m_{d}}}-\frac{\zeta_{d}}{\tau_{m_{d}}}\right)-\frac{\zeta_{s}}{\tau_{m_{d}}^{2}}
$$

Thus,

$$
\frac{\mathrm{d}^{2} \ln R_{d}}{\mathrm{~d} t^{2}}=\zeta_{d} / \tau_{m_{d}}^{2}
$$

Finally, setting $R_{e q}=R_{d}$ in Equation (47), and solving for $\zeta_{d}$, we obtain:

$$
\zeta_{d}=\frac{\zeta_{e q}+\zeta_{s} \tau / \tau_{m_{d}}}{1+\tau / \tau_{m_{d}}}
$$

This expression shows that if the evolution is much slower than the thermal relaxation ( $\tau \ll \tau_{m_{d}}$ ), the donor radius follows the equilibrium radius closely, whereas if mass transfer occurs rapidly, the donor reacts adiabatically. Equations ((34), (36), (40), and (44)) referred to as "the evolution equations".

\section{Stability Analysis of Mass Transfer}

\section{Mass Transfer Rate}

Mass transfer will proceed on a time-scale which depends critically on the changes in the radius of the donor star and that of its Roche lobe in response to the mass loss; if the star expands faster than its Roche lobe or shrinks less rapidly than its Roche lobe for a prolonged time, mass transfer will be unstable and the donor star may disintegrate. If the donor star expands less rapidly or shrinks faster than its Roche lobe, mass transfer will generally be stable and may continue for a long time.

We now introduce the concept of the equilibrium mass transfer rate, which is the mass transfer rate that a stable, semi-detached binary undergoes for a given rate of driving. The equilibrium mass transfer rate is a function of the driving rate, the consequential angular momentum loss mechanisms and the value of the mass ratio $q$. The mass transfer rate can be written generally as:

$$
\dot{m}_{d}=-\dot{m}_{0}\left(m_{1}, m_{2}, a\right) f\left(\Delta R_{d}\right)
$$

Under the assumption that $\dot{M}_{0}$ is a function of the binary parameters, whilst $f$ is a strong function of the depth of contact $\Delta R_{d}$, we can write:

$$
\ddot{m}_{d}=-\dot{m}_{0} \frac{\partial f}{\partial \Delta R_{d}}\left(\frac{\dot{R}_{d}}{R_{d}}-\frac{\dot{R}_{d}}{R_{L}}\right)
$$

For equilibrium mass transfer, we let $\ddot{m}_{d}=0$, i.e. the mass transfer rate itself does not evolve very fast. Thus, using the evolution equations and Equation (46) we have:

$$
\left(\frac{\dot{m}_{d}}{m_{d}}\right)_{e q}=\frac{v_{L}-v_{d}}{2\left(q_{\text {stable }}-q\right)}=\frac{v_{L}-v_{d}}{\zeta_{d}-\zeta_{L}}
$$

where,

$$
q_{\text {stable }}=q_{a}-\frac{\zeta_{r L}}{2}+\frac{\zeta_{d}}{2}
$$

is the critical mass ratio for stability of mass transfer. For example, for a polytropic donor with $n=3 / 2$ (which is representative of a white dwarf donor) $\zeta_{d} \sim-1 / 3, \quad \zeta_{r L} \sim 1 / 3$ and so $\left(\frac{\dot{m}_{d}}{m_{d}}\right)_{e q}=\frac{\dot{J}_{\text {sys }} / J_{\text {orb }}}{2 / 3-\zeta_{c}-q}$.

In general one can write from Equation (51). 


$$
\dot{m}_{d}=-2 \frac{m_{0}}{m_{d}} \frac{\partial f}{\partial \Delta R_{d}}\left(q_{\text {stable }}-q\right)\left(\dot{m}_{d}-\left(\dot{m}_{d}\right)_{e q}\right)
$$

when $q<q_{\text {stable }}$, the pre-factor on the right hand side of Equation (54) is negative and thus the mass transfer rate tends toward the equilibrium value implying that the mass transfer is stable. On the other hand, when $q>q_{\text {stable }}$, we can see that the system cannot reach the equilibrium value and this implies that the mass transfer is unstable. It is possible during the course of the evolution that a system that initially has $q>q_{\text {stable }}$ can evolve into a system with $q<q_{\text {stable }}$.

One of the components of the binary gets into contact with its Roche lobe either due to the expansion of the star (the donor) and/or due to shrinking of the Roche lobe as a result of angular momentum loss by GWR. In order to determine exactly when a binary with given total mass and mass ratio $q$ becomes semi-detached, one needs to specify the radii of the component stars and their corresponding Roche lobes. The condition for stability can be expressed as:

$$
\dot{R}_{d} \leq \dot{R}_{L_{d}}
$$

which simply states that the donors radius must shrink at least as fast as its Roche lobe or that the donor can expand no faster than its Roche lobe expands.

The first factor we will consider is the change in the orbital separation resulting from a mass transfer event. The orbital angular momentum, $J_{\text {orb }}$ for a binary composed of two point masses is given by Equation (2). We can use Kepler's third law:

$$
\Omega^{2} a^{2}=G M
$$

to eliminate the angular frequency from Equation (2) to obtain:

$$
J_{\text {orb }}=m_{a} m_{d} \sqrt{\frac{G a}{M}}
$$

If we logarithmically differentiate Equation (57) with respect to time and from Equation (10) with $e=0$, we obtain:

$$
\frac{\dot{J}_{\text {orb }}}{J_{\text {orb }}}=\frac{\dot{m}_{a}}{m_{a}}+\frac{\dot{m}_{d}}{m_{d}}+\frac{1}{2} \frac{\dot{a}}{a}-\frac{1}{2} \frac{\dot{M}}{M}
$$

From Equation (15), Equation (58) becomes

$$
\frac{\dot{a}}{a}=2 \frac{\dot{m}_{d}}{m_{d}}(q-1)
$$

We can then see that if the donor is the more massive star $(q>1)$, the orbital separation will shrink upon mass transfer. Conversely, if $q<1$, a will increase.

From Roche lobe geometry and following [10], the Roche lobe radius $R_{L_{d}}$ can be crudely approximated as:

$$
\frac{R_{L}}{a} \propto\left(\frac{M d}{M}\right)^{1 / 3}=\frac{2}{3^{4 / 3}}\left(\frac{m_{d}}{m_{a}+m_{d}}\right)^{1 / 3}=0.462\left(\frac{M d}{M}\right)^{1 / 3}=0.462\left(\frac{q}{1+q}\right)^{1 / 3}
$$

A more accurate approximation for the Roche lobe radius was determined by Eggleton (1983) and is given by

$$
\frac{R_{L_{d}}}{a}=\frac{0.49 q^{2 / 3}}{0.6 q^{2 / 3}+\ln (1+q)^{1 / 3}}
$$

for $0<q<\infty$ Eggleton (1983). Differentiating Equation (60) with respect to time, we obtain:

$$
\frac{\dot{R}_{L_{d}}}{R_{L_{d}}}=\frac{\dot{a}}{a}+\frac{1}{3} \frac{\dot{m}_{d}}{m_{d}}-\frac{1}{3} \frac{\dot{M}}{M}
$$

Combining Equation (62) with Equation (58) we arrive at 


$$
\frac{\dot{R}_{L_{d}}}{R_{L_{d}}}=2 \frac{\dot{J}_{\text {orb }}}{J_{\text {orb }}}-\frac{5}{3} \frac{\dot{m}_{d}}{m_{d}}+2 \frac{\dot{m}_{d}}{m_{a}}+\frac{2}{3} \frac{\dot{M}}{M}-2 \frac{\dot{m}}{m_{a}}
$$

which implies

$$
\frac{\dot{R}_{L_{d}}}{R_{L_{d}}}=2 \frac{\dot{J}_{\text {orb }}}{J_{\text {orb }}}+\left(2 \frac{m_{d}}{m_{a}}-\frac{5}{3}\right) \frac{\dot{m}_{d}}{m_{d}}+\left(\frac{2}{3}-2 \frac{m}{m_{a}}\right) \frac{\dot{M}}{M}
$$

For conservative mass transfer Equation (64) becomes

$$
\frac{\dot{R}_{L_{d}}}{R_{L_{d}}}=\left(2 \frac{m_{d}}{m_{a}}-\frac{5}{3}\right) \frac{\dot{m}_{d}}{m_{d}}
$$

which parametrized as:

$$
\frac{\dot{R}_{L_{d}}}{R_{L_{d}}}=\zeta_{d} \frac{\dot{m}_{d}}{m_{d}}
$$

In the conservative case Equation (65) indicates that if $m_{d}>\frac{5}{6} m_{a}$ the donor Roche lobe will be contract upon mass loss as $\dot{m}_{d}$ is negative.

Finally, we consider the response of the donors radius to change in its mass. For stars like the sun it is well known that the mass and radius are approximately proportional to one another. Using the expression of (66), the mass radius relation for solar type stars implies

$$
\frac{\dot{R}_{L_{d}}}{R_{L_{d}}}=\frac{\dot{m}_{d}}{m_{d}}
$$

If we substitute Equation (65) and Equation (67) into Equation (55) we obtain a limiting stable mass ratio for the binary star.

$$
q_{\text {stable }}=\frac{m_{d}}{m_{a}} \leq \frac{4}{3}
$$

If the mass ratio exceed this values the Roche lobe will be shrink faster than the star can contract and mass transfer will proceed on a dynamical time scale. If $q \leq \frac{4}{3}$ the star will, on a time scale set by the mass transfer rate detach from is Roche lobe. From Equation (64) any mechanism Schutz (1990) that removes orbital angular momentum from the binary will cause the Roche lobe of the donor to contract.

\section{Analytical and Numerical Solutions}

\subsection{Analytical Solutions}

Before we proceed upon studying the complete numerical solutions for the evolution of orbital parameters and the evolution equations we derived in Section (2) for different astrophysical scenarios, we obtain analytic solutions for the time evolution of the geometry of mass transfer rate. We can do this only on the assumption that most of the parameters characterizing the binary remain constant or evolve slowly as compared to the evolution of the mass transfer rate. [5] was among the first to attempt such analysis and following them, we generalize their results to an arbitrary polytropic index and to isothermal atmospheres. Analytic solutions are useful in providing physical insights into the expected behaviour of the binary system in the limit where the assumptions imposed to obtain the analytic solutions are valid approximately. To make Equations ((60), (61) and (68)), the two figures are plotted for different values of the mass ratio $q=m_{d} / m_{a}$ for comparison in Figure 1 and Figure 2. 
The simple form of Equation (60) is a reasonable approximation to the more accurate Eggleton [11] formula for mass ratios $q \preceq 0.9$. Thus we see that the Roche lobe radius is directly proportional to the separation for a fixed mass ratio. Given Roche geometry and the structure of the star, we can now specify the mass transfer rate. In general, we expect the mass transfer to be a strong function of the "depth of contact", defined as the amount by which the donor overflows its Roche lobe $R_{d}=R_{d}-R_{L}$ and of the structure of the star, which can be described in different ways depending on the problem at hand.

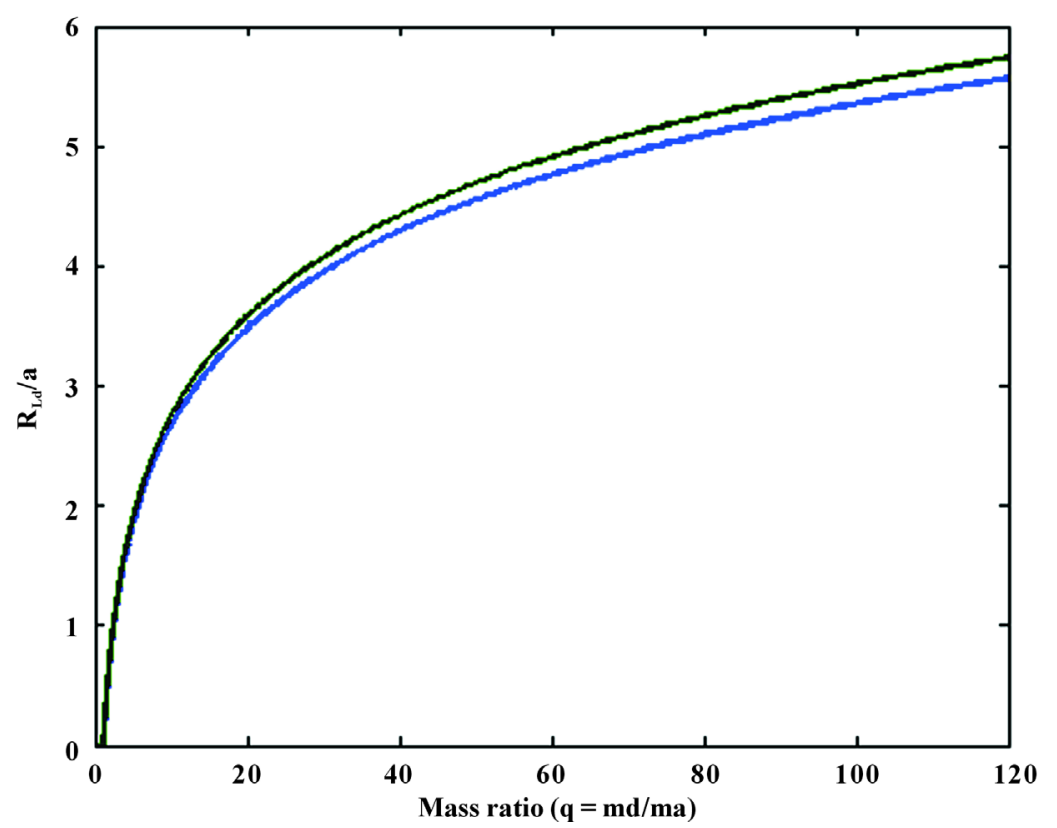

Figure 1. Comparison of [10] (Eq. (60), blue curve) and [11] (Eq. (61)) formulae for the Roche radius, normalized to the binary separation vs. mass ratio $\left(q=\frac{m_{d}}{m_{a}}\right)$.

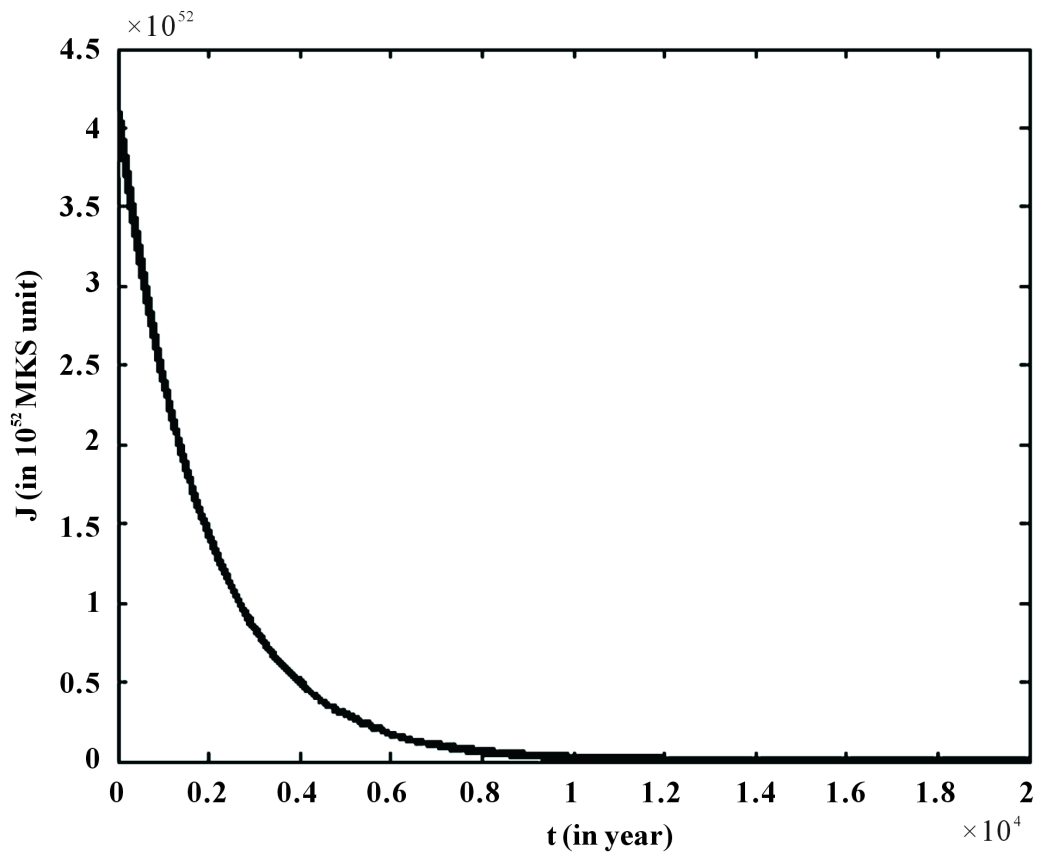

Figure 2. Change in the orbital angular momentum of the binary system with time. 
Thus if $q=\frac{m_{d}}{m_{a}} \approx 0, \dot{a}>0$ which implies that the orbit will be enlarge and if $q=\frac{m_{d}}{m_{a}} \approx \infty, \dot{a}<0$ that the orbit will be shirk. This means the radius of a Roche lobe filling star depends only on the binary separation and the mass ratio. The blue curve shows that the stable conservative mass transfer while the green one is the unstable mass transfer.

In Figure 2, we have plotted the equilibrium angular momentum values for a range of binary system with a time and for a mass ratio $q=1$, taking the mass of the black hole $M_{B H}=1.75 M_{\odot}$. We here produce analytical solutions for the non conservative mass transfer in close binary system taking the initial masses of the primary and secondary as $m_{a}=2.5 M_{\odot}$ and $m_{d}=5 M_{\odot}$.

The change in mass for both the accretion and donor as well as the changing of the orbital angular frequency with time are derived in Equation (21) within the pre-assigned time scale of mass exchange as $t=10^{4}$ (years) and period $p=100$ (days), the orbital angular momentum $J$ continues to show a steady decreasing with the increasing time in Figure 3 and Figure 4.

Considering an interacting double degenerate system with initial masses of donor and accretor $m_{a}=0.24 M_{\odot}$ and $m_{d}=0.65 M_{\odot}$ (dotted line) and a "semi-degenerate" low-mass helium star donor plus white dwarf accretor of the same initial masses (dash or blue line), and the periods $(P=2,3,4)$ from Roche lobe overflow; lower limits of the disk is equilibrium instability region according to [12] for $q=0.5$ and conventional evolutionary computations may be not adequate for description of mass transfer process.

From Equation (18), the change of orbital period is proportional to the change in $M_{\text {dot }}$. Prolonged, conservative mass transfer is obtained when the initial mass of donor star is more massive, and the period decreases; hence star move close together.

\subsection{The Binary Parameters}

It is worthwhile to describe the binary parameters used by [4] [5] in their pioneering analysis in some detail, since we are using it as a comparison to our numerical solutions in Section (4.4). We shall thus obtain analytic as well as numerical solutions for a general polytropic index $n$ to compare our results with those obtained from a full numerical evolution. The mass transfer rate for a polytropic donor is given by Jedrzejec (1969) assuming laminar flow, quoted by Paczynski \& Sienkiewicz (1972) and from Equations. ((51)-(54)) we obtain:

$$
\dot{m}_{d}=-\dot{M}_{0}\left(\frac{R_{d}-R_{L_{d}}}{R_{d}}\right)^{n+3 / 2}
$$

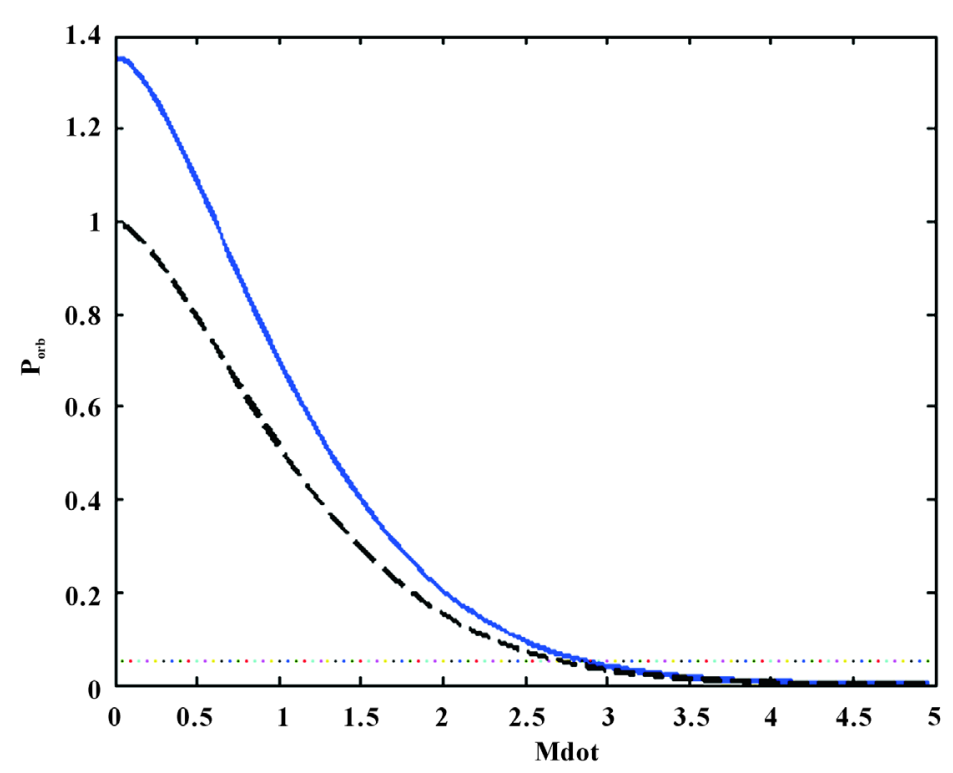

Figure 3. Orbital period ( $P_{\text {orb }}$ ) versus $M_{\text {dot }}$ for “typical” AM CVn-stars. 


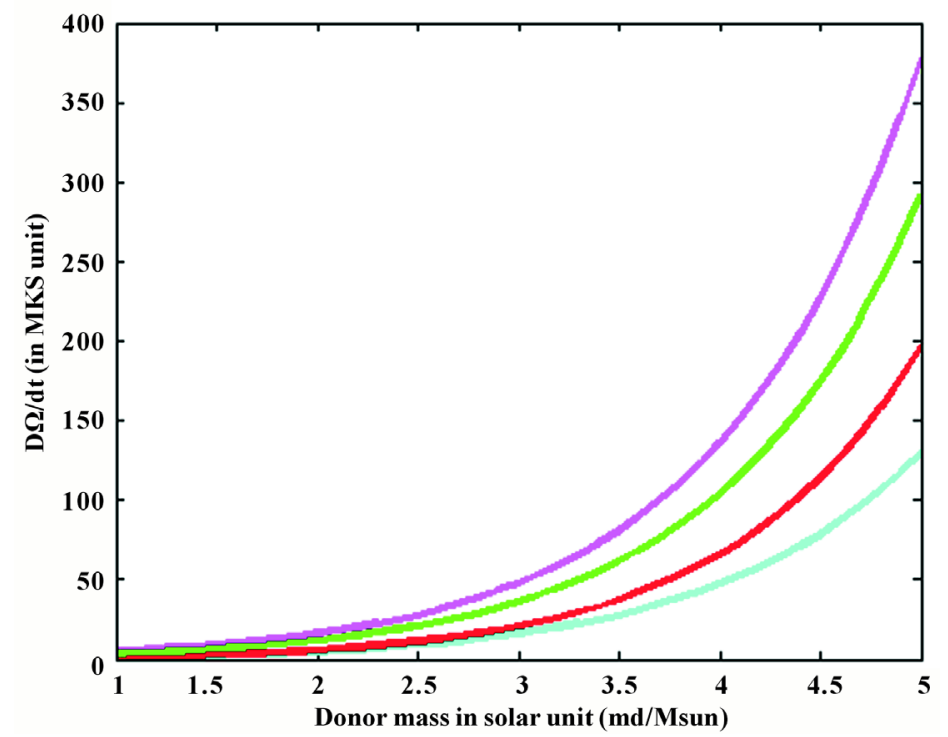

Figure 4. The total rate of change of the orbital angular frequency of the binary (Eq. (30)) related to the mass lost from the system of two point masses which will coalesce due to gravitational wave emission in a time interval shorter than $10^{10} \mathrm{yr}$, as a function of the donor mass in solar unit $\left(m_{d} / M_{\odot}\right)$. As we go from top to bottom on the right side; the lines (curves) are calculated for magenta $\left(10 M_{\odot}+10 M_{\odot}(B H+B H)\right)$ which corresponds to an initial $1 M_{\odot}$ donor whose central hydrogen abundance is 0.005 at the onset of mass transfer, green $\left(10 M_{\odot}+1.4 M_{\odot}(B H+N S)\right)$ corresponds to a donor whose central hydrogen abundance has just reached zero at the onset of mass transfer, the red curve (line) is the analytical value of the rate of change of the orbital angular frequency versus donor mass in solar unit (the analytical value of those compact objects) and cyan $\left(1.4 M_{\odot}+1.4 M_{\odot}(N S+N S)\right)$ corresponds to the case for which the donor has developed a $0.005 M_{\odot}$ helium core.

Raising both sides of the Equation (69) to the power $2 /(2 n+3)$ and differentiating, we obtain:

$$
\frac{\mathrm{d}}{\mathrm{d} t}\left(\dot{m}_{d}\right)^{2 / 2 n+3}=-\left(\dot{M}_{0}\right)^{2 / 2 n+3}\left[\left(v_{d}-v_{L d}\right)+\frac{\dot{m}_{d}}{m_{d}}\left(\zeta_{d}-\zeta_{L d}\right)\right]
$$

where we have set the factor $R_{L} / R_{d}$ to unity, given that in most situations $\Delta R_{d} \ll R_{d}$.

Defining a positive dimensionless mass transfer $X=\left(\dot{m}_{d} / M_{0}\right)^{2 /(2 n+3)}$, and a characteristic time scale $\tau=\dot{m}_{d} / \dot{M}_{0}$, Equation (70) becomes

$$
\frac{\mathrm{d} X}{\mathrm{~d} t}=-\frac{\zeta_{d}-\zeta_{L d}}{\tau} X^{n+3 / 2}
$$

The solution can be easily inverted to yield

$$
X(t)=X(0)\left[1+X(0)^{n+1 / 2}(n+1 / 2)\left(\zeta_{d}-\zeta_{L d}\right) t / \tau\right]^{-2 / 2 n+1}
$$

where $X(0)$ is the initial mass transfer rate, normalized as Equation (72).

Returning now to Equation (70), with the same definitions as Equation (72) for $X$ and $\tau$, we obtain for the general case in which driving is present

$$
\frac{\mathrm{d} X}{\mathrm{~d} t}=-\frac{\zeta_{d}-\zeta_{L d}}{\tau}\left(X^{n+3 / 2}-X_{e q}^{n+3 / 2}\right)
$$


where $X_{e q}^{n+3 / 2} \equiv-\left(\dot{m}_{d} / \dot{M}_{0}\right)=\left(v_{d}-v_{L d}\right)\left(\tau / \zeta_{d}-\zeta_{L d}\right)$ is the equilibrium value normalized to $M_{0}$. Note that in the stable case, this value is positive; while it is negative in the unstable case. Before we attempt to solve the above differential equation, it is clear from its form and the signs just discussed, that it describes a stable solution in which $X \rightarrow X_{e q}$ when $q<q_{\text {stable }}$. if, however, $q>q_{\text {stable }}$, the right had side of Equation (73) is positive even if the mass transfer vanishes initially, and it just gets bigger as the mass transfer grows. Since $X$ diverges for the non driving case in a finite time, the driven case diverges even sooner.

Considering Equation (70) again we define $X^{*}=\left[\dot{m}_{d} /\left(\dot{m}_{d}\right)_{e q}\right]^{2 / 2 n+3}$, and $1 / \tau^{*}=\left(v_{d}-v_{L d}\right)\left(\dot{m}_{d} /\left(\dot{m}_{d}\right)_{e q}\right)^{2 / 2 n+3}$.

The differential equation for the evolution of mass transfer now becomes

$$
\frac{\mathrm{d} X^{*}}{\mathrm{~d} t}=\left(X^{*}\right) \frac{1}{\tau^{*}}\left(1-\left(X^{*}\right)\right)\left|X^{*}\right|^{n+3 / 2}
$$

Thus, for the stable case ( $X^{*}>0$, while $\left(X^{*}<0\right.$ for the unstable case, and $\tau^{*}$ is defined positive). The general analytic solution comprising both the stable and the unstable case can be given in terms of the hyper geometric function, as follows:

$$
\frac{t}{\tau^{*}}=X_{d}^{*}\left(1, \frac{1}{n+3 / 2} ; 1+\frac{1}{n+3 / 2},\left(X^{*}\right)\left|X^{*}\right|^{n+3 / 2}\right)
$$

Though it is not possible to invert this general solution to obtain the mass transfer rate $X$ as a function of time, particular solutions for specific values of $n$ can be inverted to obtain simple solutions. For example, $n=3 / 2$ yields the [5] solution:

$$
\frac{t}{\tau}=-\frac{1}{2}\left[\frac{(1-X)^{3}}{1-X^{3}}\right]+\sqrt{3}\left[\arctan \left(\frac{2 X+1}{\sqrt{3}}\right)-\frac{\pi}{6}\right]
$$

where, $X=\left(-\dot{m}_{d} / \mu\right)^{1 / 3}, \tau=1 / 3\left[\dot{m}_{0} / m_{d}\left(\zeta_{d}-\zeta_{L d}\right)\left(v_{d}-v_{L d}\right)\right]^{-1 / 3}$.

Likewise, $n=1 / 2$ yields $X^{*}=\tan \left(t / \tau^{*}\right), \quad X^{*}<0$ unstable and $X^{*}=\tanh \left(t / \tau^{*}\right), \quad X^{*}>0$ stable.

\subsection{Isothermal Atmospheres}

In case of isothermal atmospheres, the mass transfer rate [13] [14] is given by

$$
\dot{m}_{d}=-m_{0} \mathrm{e}^{\left(\left(R_{d}-L_{d}\right) / \alpha\right)}
$$

where, $\alpha$ is the scale height. This form of the mass transfer equation is much simpler to integrate than the one for the polytropes considered above. With the same approximations and notation as in the steps leading to Equation (73), and defining $X=-\dot{m}_{d} / \dot{m}_{0}=\mathrm{e}^{\left(\left(R_{d}-R_{L}\right) / \alpha\right)}$, we obtain:

$$
\frac{1}{X} \frac{\mathrm{d} X}{\mathrm{~d} t}=-\frac{\zeta_{d}-\zeta_{L}}{\tau} \frac{R_{d}}{\alpha}\left(X-X_{e q}\right)
$$

This is easily integrable and invertible to get the following:

$$
X=\frac{X_{e q}}{1-\left(\left(1-X_{e q}\right) / X_{0}\right) \mathrm{e}^{\tau / \tau_{\text {iso }}}}
$$

where $\tau_{\text {iso }} \equiv \alpha / R_{d}\left(v_{d}-v_{L}\right)$ is the time scale required for the driving to change the depth of contact by $\sim \alpha$, and $X_{0}$ is the initial value, always positive for physically meaningful cases. In the stable case $X_{\text {eq }}>0$, and $X \rightarrow X_{e q}$, while $X_{e q}<0$ for the unstable case and $X$ diverges in a finite time $t_{d i v}=\tau_{\text {iso }} \ln \left(1-X_{e q} / X_{0}\right)$. If no driving is present, we may set $X_{e q}=0$ and integrate Equation (78) for an isothermal donor.

The result is again simple and instructive.

$$
X=\frac{X_{0}}{1+\left(\zeta_{d}-\zeta_{L}\right) X_{0} \frac{R_{d}}{\alpha} \frac{t}{\tau}}
$$


In the stable case, for any initial mass transfer, the system will detach and mass transfer will tend to zero. In the unstable case, any non-zero initial mass transfer will grow and diverge in a finite time.

We specify the parameters of the binary at its initial state; $m_{a}=0.62 M_{\odot}, m_{d}=1.1 M_{\odot}, R_{a}=0.012 R_{\odot}$, $R_{d}=0.0072 R_{\odot}$, and $a=0.037 R_{\odot}$ and for the binary, it turns out that the adiabatic coefficients $\zeta_{d} \sim-0.54$ and $\zeta_{L_{d}} \sim-0.38$ are related to Equations ((72), (73), (75), and (76)). We notice that since $\left|\zeta_{d}\right|>\left|\zeta_{L_{d}}\right|$; the radius of the star increases at a rate faster than the Roche lobe radius, and the resulting mass transfer is unstable.

The analytic solutions, both the stable and the unstable, are shown in Figure 5.

Note that initially, the stable and the unstable solutions in Figure 5 almost exactly overlap. This is true as long as the system is either detached or the depth of contact is relatively small. As soon as the binary evolves to deeper contact, the unstable solution diverges rapidly and blows up in a finite time. This outcome is predicted by the unstable analytic solution which has been obtained by assuming that the basic primary parameters: the mass ratio $q$, driving rate $v_{L}$ and the masses of the components do not change during the evolution. This is obviously not true, especially in the unstable case, since by definition in the unstable case these parameters are evolving rather rapidly (Figure 5).

\subsection{Numerical Solutions}

In Section (4.2 and 4.3), we have derived the basic evolution equations; Equations ((72), (73), (75), and (76)) from Equations ((51)-(54)) in Section (3.1) that for the numerical results and the corresponding analytic solutions for different cases, under certain restrictive approximations. These analytic solutions are a useful reference to compare our numerical results to, since in the limit that the assumptions used to derive the analytic solution are met, the numerical solutions should approach the analytic ones. However, in general, the analytic solutions cannot explain the behavior of a given system accurately and especially when the system evolves rapidly, solving the evolution equations numerically leads to a different outcome than what one would expect analytically.

\section{Limitations of the Analytic Solutions}

The analytic solutions of equations in Section (4.2 and 4.3) from Section (2.4) and through Section (3.1) are obtained under the following assumptions:

1) The driving rate given by $v_{L}$ is constant throughout the evolution.

2) The separation " $a$ " of the binary is effectively constant even though the mass transfer rate changes, which is not true for " $a$ " "real system" (see Equation (36)).

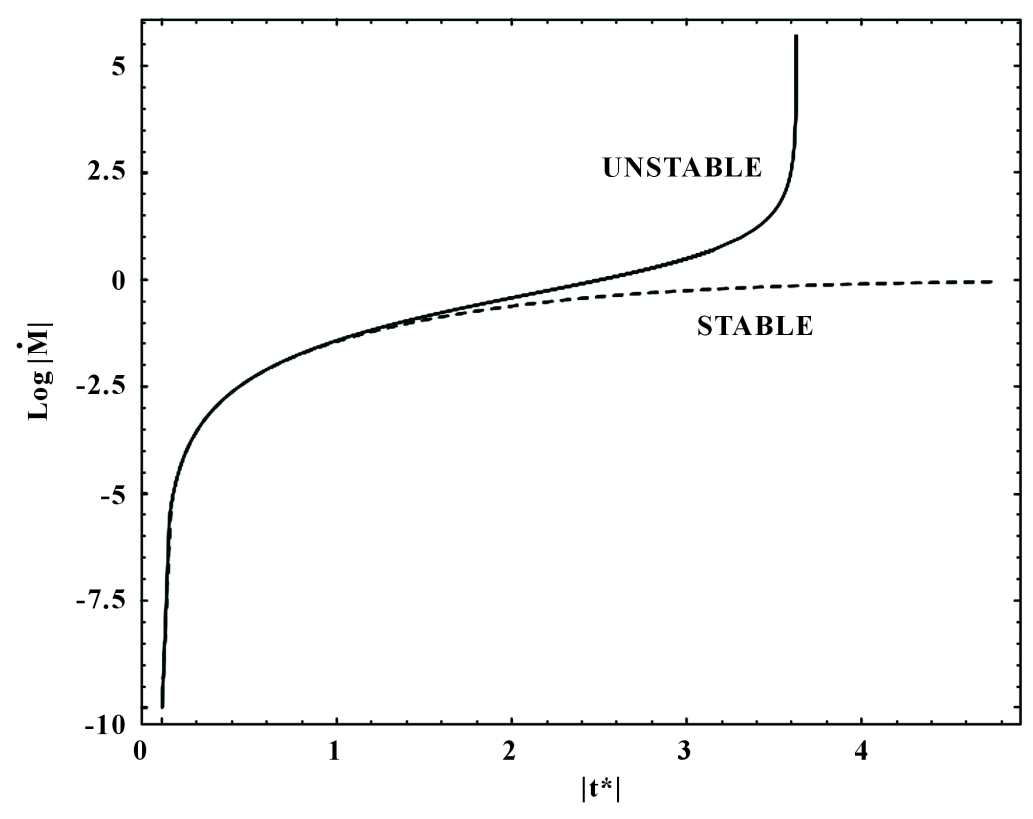

Figure 5. The solutions for the stable (dotted line) and unstable cases. 
3) The tidal effects are effectively ignored.

4) A system that is initially unstable ( $q>q_{\text {stable }}$ ), remains so throughout the evolution, since $q$ is assumed to be the same throughout and it is possible for an initially unstable system to evolve to a stable configuration.

In order to overcome some of these shortcomings one has to numerically integrate the evolution equations in a self consistent way. In particular, in what follows, we allow for the changes in the masses of the components assuming conservative mass transfer (and hence the mass ratio $q$ ), allow the binary separation to change as a result of any driving present, and compute the values of $\zeta_{d}$ and $\zeta_{L}$ as they evolve. The values of $\zeta_{d}$ depend on the adopted mass-radius relationship for the donor. To calculate $\zeta_{L}$, we need to specify how the mass and angular momentum are redistributed in the binary during mass transfer which depends on the particular case at hand. For example, it depends on whether the stream impacts the accretor or if an accretion disk is present; if the mass transfer is sub-critical and conservative, or if mass and angular momentum are being ejected from the system following supercritical mass transfer (Figures 6-8).

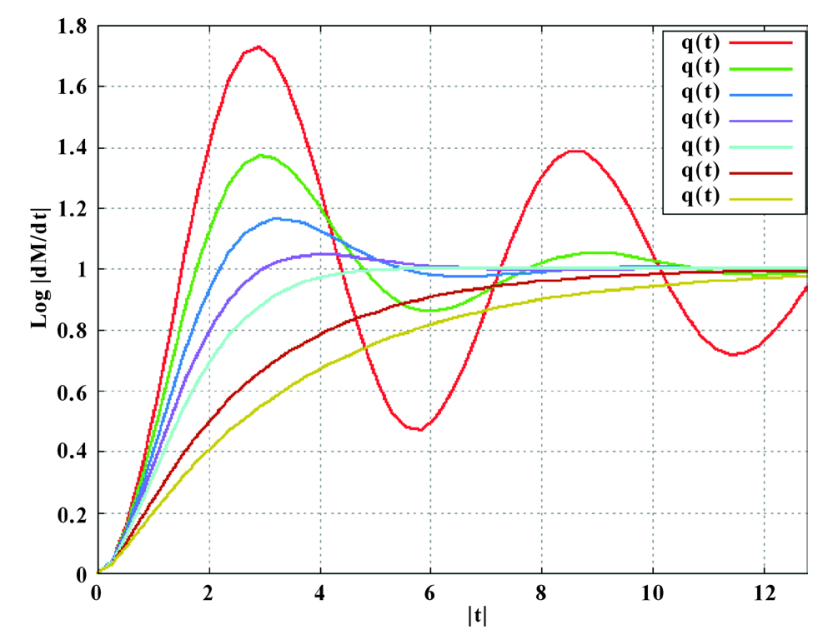

Figure 6. Numerical and Analytic Solutions for Polytropes. Comparison of numerical integrations with the analytic solution in Section (4.2). The mass transfer rate in solar mass normalized to the initial equilibrium rate as a function of time in units of the initial $\tau$ : analytic (red curve) and numericalgreen $(q=0.985)$, blue $(q=0.875)$, magenta $(q=0.816)$, cyan $(q=0.656$ same as [5]), Darkred $(q=0.625)$ and orange $(q=0.416)$.

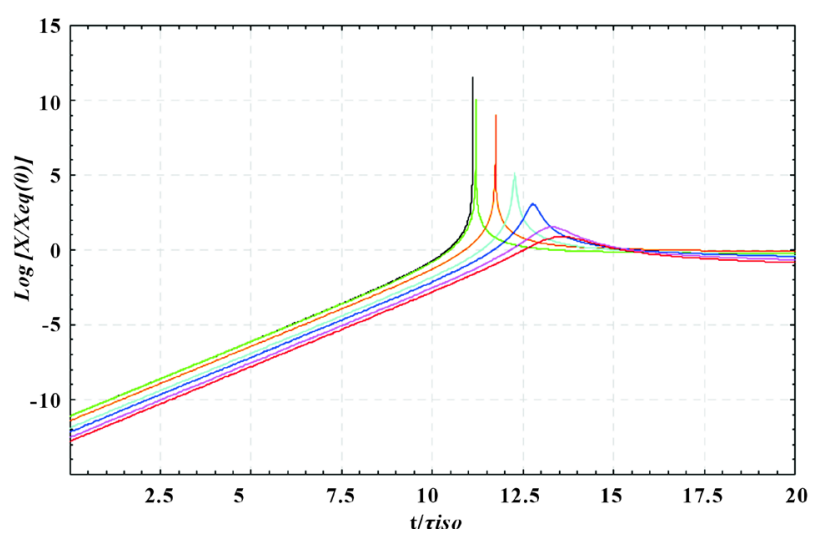

Figure 7. Comparison of numerical integrations with the analytic solutions for isothermal atmospheres. The natural logarithm of the mass transfer rate normalized to the initial equilibrium rate for the case with $q=0.663$, is shown as a function of time in units of $\tau_{\text {iso }}$ : analytic (black curve) and numerical green ( $q=0.985)$, orange $(0.613)$, cyan $(q=0.656$ same as [5]), blue (0.875), magenta (0.816) and red (0.416). 


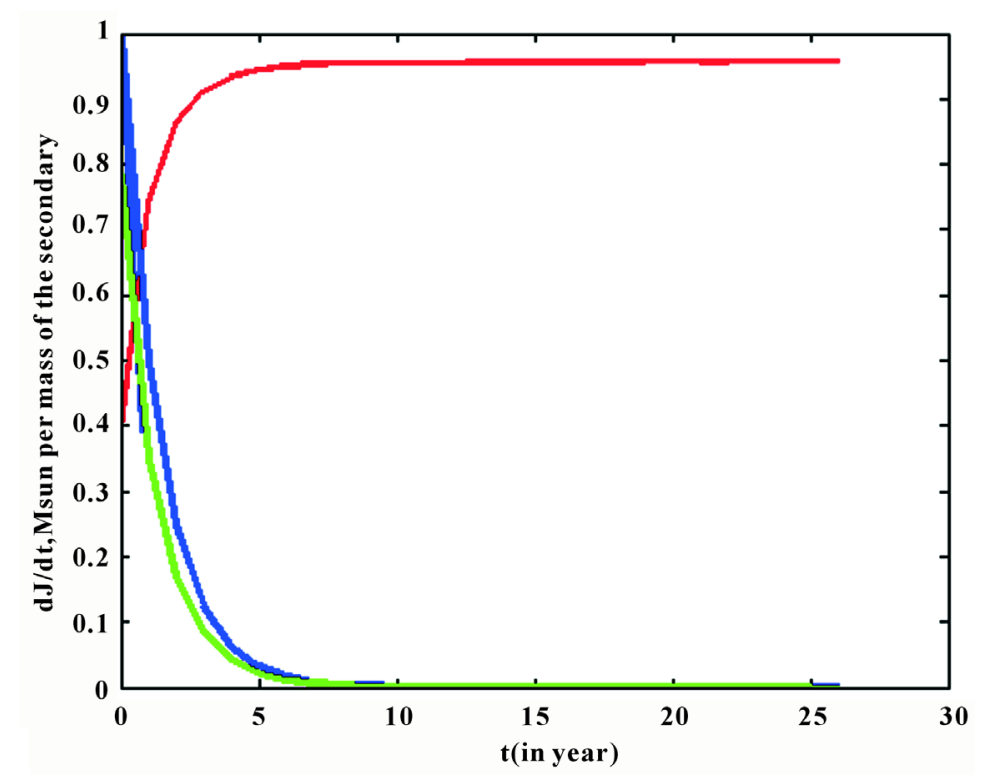

Figure 8. Loss of orbital angular momentum and decrease in the mass of the secondary with time (in year).

We simulate exactly the same initial conditions as the ones assumed to derive the analytic solution, a constant driving rate and the same mass-radius relationship. We then relax the constraints and let the system evolve in a self-consistent manner for different values of the mass ratio $q$. We observe that initially all the curves overlap, but as the depth of contact increases, the mass transfer rate increases. As the system evolves, the orbital separation, which is decreasing initially, begins to increase as per Equation (36) which in turn decreases the driving rate. At some point during the evolution $q$ becomes less than $q_{\text {stable }}$ and the system evolves to stable mass transfer rates. From our analysis in Section (4.2), we expect the systems with $q>q_{\text {stable }}$ to be unstable, and the higher the mass ratio, the more unstable the system is. This is indeed what we observe in the numerical solutions. The numerical solutions reach a peak in the mass transfer rate in a finite time, but turn around and a stable mass transfer is established. In any case, our results suggest and demonstrate that at least some of the binaries that start off unstable do survive the mass transfer instability and evolve into systems like AM CVn-evolving to higher separations and diminishing mass transfer rates. This result, which is not predicted by the analytic solution, has important consequences for population synthesis, understanding AM CVn type systems.

In Figure 7, we plot the analytic and numerical solutions for an isothermal donor for different values of the mass ratio. Unlike the polytropic donor case, which has a fixed reference point where the donor fills its Roche lobe ( $R_{d}=R_{L}$ ), the isothermal case does not have a fixed reference point to which we can normalize our results. We, rather arbitrarily start the integrations when the depth of contact is $R_{d}-R_{L}=-11.5 \alpha$, corresponding to an initial mass transfer rate of $10^{-5}$ of the reference rate. The behavior of the systems is qualitatively the same as the polytropic donors. Note that the y-axis in Figure 7 is the natural logarithm of $\mathrm{x}$ which from Section (4.3), is just the depth of contact $R_{d}-R_{L}$ in units of the pressure scale height $\alpha$, normalized to the reference depth corresponding to the equilibrium rate for $q=0.663$.

Thus we here produce a numerical analysis for the rate of change of orbital angular momentum(green line), the rate of change of mass of the secondary star (blue line), and Increase in the mass of the primary with time (in year) in a close binary system taking the initial masses of the primary as $m_{a}=2.5 M_{\odot}$ and $m_{d}=5 M_{\odot}$. For the present calculation, we consider the values of the parameters as $a=2.78, p=30$ (per year) and $q=0.8$. But afterwards as the mass accretion rate by the primary varies slowly and consequently $m_{a}$ shows a very weak variation with time, the orbital angular momentum $J_{\text {orb }}$ continues to slow as steady decreasing with the decreasing in $m_{d}$.

\section{Conclusions}

We have presented a simple, analytical and numerical approach to describe the mass transfer in the evolution of 
binary star systems in which we track the mass of each component, the total mass of the system, the orbital angular momentum and the spin angular momenta of each component. We have included the effects of mass and angular momentum loss from the system and also the exchange of mass and angular momentum between the components of the binary.

The main reason why many binary systems transfer matter at some stage of their evolutionary lifetimes is during the system evolution. One of the stars in a binary system may increase in radius filling its Roche lobe, or the binary separation may shrink because orbital angular momentum is being lost from the system as a consequence of stellar wind mass loss, or gravitational radiation.

There are two general mechanisms for the transferring angular momentum out of the system: First, binary systems emit gravitational radiation, which carries away angular momentum. The rate of angular momentum loss increases as the orbital separation decreases, but it decreases as the total mass of the system decreases. Because both the secondary mass and the orbital separation decrease as CV systems evolving, the net effect is a loss mechanism that does not depend strongly on the orbital period.

Our investigation indicates that the stability of the mass transfer processes depends on: how the radius of the donor star responds to the mass loss, how the orbit responds to mass transfer, and how the mass-gainer responds to the matter that is being dumped on it.

The Roche lobe of the mass donor shrinks as a consequence of its mass loss, increasing the rate at which it loses mass is referred to unstable mass transfer and the Roche lobe of the mass donor grows as a consequence of its mass loss, stopping the mass transfer is referred to stable mass transfer.

In this paper we have made an effort to find a numerical and analytical solutions for conservative and non-conservative mass transfer in close binary systems which can address the gradually reducing rate of the mass accretion by the accretor from the donor with respect to time as well as with respect to the increase in mass of the accretor [15]. In view of this we have presented the time dependent profiles of the masses of both the component stars in a close binary as well as the orbital angular momentum of the system. Our analytical solutions shows for the non-conservative mass transfer in close binary system taking the initial masses of the primary and secondary as $m_{a}=2.5 M_{\odot}$ and $m_{d}=5 M_{\odot}$ with $t=10^{4}$ (in years) and $p=100$ (in days), and $10 M_{\odot}+10 M_{\odot}(B H+B H)$ which corresponds to an initial $1 M_{\odot}$ donor whose central hydrogen abundance is 0.005 at the onset of mass transfer, $10 M_{\odot}+1.4 M_{\odot}(B H+N S)$ corresponds to a donor whose central hydrogen abundance has just reached zero at the onset of mass transfer and $1.4 M_{\odot}+1.4 M_{\odot}(N S+N S)$ corresponds to the case for which the donor has developed a $0.005 M_{\odot}$ helium core (see Figure 4), the orbital angular momentum $J$ continues to show a steady decreasing with the increasing time (see Figure 2).

In conservative mass transfer and from Equation (15); if $m_{a}$ is the mass losing star, $\dot{m}_{a}$ will be negative, therefore we conclude that if the mass donor star is more massive, then the orbit will shrink and hence period decreases. But if the mass donor is less massive than the accretor component then orbit will be widened and period increases.

We have also presented a new finding (analytic and numeric solutions) for the evolution of binary systems, generalizing the results of [4] to binaries with any polytropic index and to binaries with isothermal atmospheres. The analytic solutions always predict that if a binary attains contact when the mass ratio $q$ is such that the ensuing mass transfer is unstable, the binary merges in a finite time. On the other hand, the numerical solutions predict that a binary that undergoes unstable mass transfer at initial contact can evolve to a state of stable mass transfer, survive and evolve into an AM CVn type system. Moreover, the analytic solutions also discussed here assume that the driving rates $v_{d}$ and $v_{L}$ in Equation (70) remain constant while the depth of contact changes. This is only approximately true; and a self-consistent solution will require numerical integrations. This is a situation we encounter in some large-scale hydrodynamic simulations of mass transfer in poly tropic binaries. We note that from Equations ((54), and (72)) in the stable case, $\zeta_{d}>\zeta_{L}\left(q<q_{\text {stable }}\right)$, the mass transfer decays asymptotically to zero over a characteristic time $\tau_{c h r}=\tau /\left[(n+1 / 2) y(0)^{n+1 / 2}\left(\zeta_{d}-\zeta_{L}\right)\right]$, it will blow up in finite time equal to $\tau_{c h r}$. Thus, the essence of the stability of mass transfer in a binary is already contained in the simple case of no driving. The presence of driving exacerbates the natural instability or, in the stable case, it settles asymptotically to a non-zero stable mass transfer, which we have been observed in AM CVns, CVs and LMXBs. In our study numerical solutions indicates more physical descriptions than analytical (Figures 1-5) one as shown in Figures 6-8.

Finally, the most useful thing, is that if one assumes only accretion and wind-like mass transfer, then most 
every binary in which mass is transferred from the less massive star is stable on both dynamical and thermal time scales. If the mass donor has a radiative envelope (not treated here), it will shrink in response to mass loss, and lose mass stable. If the donor has a convective envelope, a modestly sized core will stabilize it sufficiently to prevent mass loss on the dynamical time scale.

\section{Acknowledgements}

We thank Entoto Observatory and Research Center for supporting this research. Negu, thanks Jigjiga University for giving study leave. This research has made use of NASA's Astrophysical Data System.

\section{References}

[1] Shore, S.N. (1994) Observations and Physical Processes in Interacting Binaries. Springer, Berlin Heidelberg, 1-133. http://dx.doi.org/10.1007/3-540-31626-4_1

[2] Soberman, G.E., Phinney, E.S. and van den Heuvel, E.P.J. (1997) Stability Criteria for Mass Transfer in Binary Stellar Evolution. Astronomy and Astrophysics, 327, 620-635.

[3] Konstantin, A.P. (2014) The Evolution of Compact Binary Star Systems. Living Reviews in Relativity, 17, 3.

[4] Pennington, R.(1985) Interacting Binary Stars. Pringle, J.E. and Wade, R.A., Eds., Cambridge Astrophysics Series, Cambridge University Press, Cambridge, 197-199.

[5] Hjellming, M.S. and Webbink, R.F. (1987) Thresholds for rapid mass transfer in binary systems. I-Polytropic Models. The Astrophysical Journal, 318, 794-808. http://dx.doi.org/10.1086/165412

[6] Ruderman, M., Shaham, J. and Tavani, M. (1989) Accretion Powered by Compact Binaries. The Astrophysical Journal, 3, 507.

[7] Tessema, S.B. (2014) Stability of Accretion Discs around Magnetized Stars. International Journal of Astronomy and Astrophysics, 4, 319-331. http://dx.doi.org/10.4236/ijaa.2014.42026

[8] Lightman, A.P. and Eardley, D.M. (1974) Black Holes in Binary Systems: Instability of Disc Accretion. The Astrophysical Journal, 187, L1.

[9] Gharami, P., Ghosh, K. and Rahaman, F. (2014) A Theoretical Model of Non-Conservative Mass Transfer with NonUniform Mass Accretion Rate in Close Binary Stars. General Relativity and Quantum Cosmology, 366, 1511.

[10] Plavec, A. and Paczynski, B. (1971) Numerical Simulations of Dynamical Mass Transfer in Binaries. Annual Review of Astronomy and Astrophysics, 9, 183.

[11] Lajoie, C.-P. and Sills, A. (2010) Mass Transfer in Binary Stars Using Smoothed Particle hydrodynamics. II. Eccentric Binaries. The Astrophysical Journal, 726, 13 p.

[12] Tsugawa, M. and Osaki, Y. (1997) Disk Instability Model for the AM Canum Venaticorum Stars. PASJ: Publ. Astron. Soc. Japan, Vol. 49, 75-84.

[13] Paczynski, B. and Sienkiewicz, R. (1972) Mass Transfer Effects in Binary Star Evolution. Acta Astronautica, 22, 73.

[14] Vayujeet, G. (2007) Mass Transfer and Evolution of Compact Binary Stars. Astronomy \& Astrophysics, $202,93$.

[15] Izzard, R.G., de Mink, S.E., Onno, RP., Langer, N., Sana, H. and de Koter, A. (2013) Massive Binary Stars and SelfEnrichment of Globular Clusters. Memorie della Società Astronomica Italiana, 1, 1-4. 